\title{
Bur open Long-term clinical results after cataract surgery with and without capsular tension ring in patients with retinitis pigmentosa: a retrospective study
}

To cite: Bayyoud T, BartzSchmidt KU, Yoeruek E. Long-term clinical results after cataract surgery with and without capsular tension ring in patients with retinitis pigmentosa: a retrospective study. BMJ Open 2013;3: e002616. doi:10.1136/ bmjopen-2013-002616

- Prepublication history for this paper are available online. To view these files please visit the journal online (http://dx.doi.org/10.1136/ bmjopen-2013-002616)

Received 20 January 2013 Revised 3 March 2013 Accepted 19 March 2013

This final article is available for use under the terms of the Creative Commons Attribution Non-Commercial 2.0 Licence; see http://bmjopen.bmj.com

Department of Ophthalmology, EberhardKarls University Tuebingen, Tuebingen, Germany

Correspondence to

Tarek Bayyoud;

tarek_bayyoud@gmx.de

\section{ABSTRACT}

Objectives: To describe the long-term clinical outcomes after cataract surgery with and without capsular tension ring (CTR) in a group of patients with retinitis pigmentosa (RP).

Design: A retrospective study.

Setting: Tertiary referral centre.

Participants: 52 eyes (46 patients) with RP. Interventions: Cataract surgery was undertaken between October 2002 and May 2010.

Primary and secondary outcome measures: Visual acuity, secondary cataract, capsular contraction syndrome (CCS), intraocular pressure, cystoid macular edema (CME), intraocular lens dislocation and endophthalmitis.

Results: The mean age at surgery was about 53 years and the overall mean follow-up was 26 months (range 360 months). The mean preoperative logarithm of the minimal angle of resolution of the best corrected visual acuity (LogMAR BCVA) in the entire group was 1.45 \pm 0.85 (95\% Cl 1.21 to 1.69$)$ and had increased to 1.32 $\pm 0.95(95 \% \mathrm{Cl} 1.06$ to $1.58, \mathrm{p}=0.02)$. The mean preoperative and the mean postoperative LogMAR BCVA in the non-CTR group (group 1) improved from 1.16 \pm 0.8 (95\% Cl 0.83 to 1.48$)$ to $0.98 \pm 0.88(95 \% \mathrm{Cl} 0.62$ to $1.33, \mathrm{p}=0.02$ ) and in the CTR group (group 2) from 1.74 $\pm 0.81(95 \% \mathrm{Cl} 1.42$ to 2.07$)$ to $1.66 \pm 0.90(95 \% \mathrm{Cl} 1.3$ to $2.03, p=0.31$ ), respectively. Secondary cataract was observed in a total of 23 eyes $(44 \%)$, of which $13(50 \%)$ were belonged to group 1 and $10(38 \%)$ to group 2. CCS was seen in a total of two eyes (4\%) all under group 1. CME was noted in two eyes $(4 \%)$, of which one belonged to group 1 and a second one to group 2. Endophthalmitis was not observed in any group. Conclusions: Both surgical approaches were beneficial to the RP patients. Eyes under group 2 showed less longterm postoperative complications. This includes secondary cataract and CCS. Eyes under group 1 performed significantly better in respect of visual acuity. Further research would include insights into the genetic subsets.

\section{INTRODUCTION}

The development of cataract is a well-known complication seen in patients with retinitis

\section{ARTICLE SUMMARY}

Article focus

- Long-term clinical outcomes after cataract surgery with and without capsular tension ring in a group of patients with retinitis pigmentosa.

Key messages

- Both surgical approaches were beneficial to patients with retinitis pigmentosa.

- Surgery with capsular tension ring implantation resulted in fewer long-term postoperative complications including secondary cataract and capsular contraction syndrome.

Strengths and limitations of this study

- Relatively small sample size of this special subset of cataract patients.

pigmentosa (RP). ${ }^{1-4}$ Treatment essentially involves phacoemulsification of the natural lens and the replacement by an artificial one. Relatively minor lens opacities may cause disproportionate functional symptoms, keeping in mind the inferior retinal function. In general, RP is an important cause of blindness among working-age people. ${ }^{1}$ Cataract surgery led to an increase of contrast and helped this patient group gain visual acuity. Cut-off filters may be used to improve contrast sensitivity. ${ }^{2}$ Although, a steady decrease is anticipated in the longterm perspective, surgical intervention is recommended, necessary to improve quality of life. Some different types of surgical add-ons have been described in the literature to approach the potential postoperative complications, one of them being the capsular contraction syndrome (CCS). ${ }^{5}$ Whether to utilise them remains to the surgeon's preference. This retrospective study tried to compare the development of short-term and long-term postoperative complications and the best corrected visual acuity (BCVA) between two different groups. Group 1 
received phacoemulsification and the implantation of an intraocular lens (IOL, type 1 surgery). In group 2 a capsular tension ring (CTR) was added to the inserted IOL (type 2 surgery). The postoperative complications taken into consideration were intraocular pressure (IOP), cystoid macular oedema (CME), secondary cataract and CCS. The former two may be treated medically, whereas the latter two ask for surgical intervention.

\section{PATIENTS AND METHODS}

The data of 46 patients with RP who had cataract removal and phacoemulsification with or without subsequent CTR implantation were retrospectively reviewed. A research was conducted according to the tenets of the Declaration of Helsinki and with the approval of the ethics committee of Tuebingen. The first group (26 eyes) had phacoemulsification and IOL implantation. In the second group (26 eyes) a CTR was additionally inserted. The surgical technique involved temporally localised clear corneal incision with $2.75 \mathrm{~mm}$ of wound size. The experienced surgeons operated this kind of demanding cataract with the risk of zonulolysis using the stop and chop technique in advanced nuclear opacity and the chip and flip technique in soft cataracts. Excessive cleaning of the posterior capsule, the equatorial bag and the anterior undersurface was performed in all cases. The IOL types used were all hydrophobic, acrylic lenses made up of a single piece or multipiece. In group 1, 1 and 25 have three-piece or one-piece IOLs, respectively. In group 2, 10 and 16 have threepiece or one-piece IOLs, respectively. The CTR type was either Croma-Pharma GmbH (Leobendorf, Austria) or Morcher GmbH (Stuttgart, Germany) fabricate of $11 \mathrm{~mm}$. There were 19 emmetropic, 6 myopic and 27 hyperopic eyes. Ophthalmological examinations were performed prior to surgery and at the follow-up visits. Examinations included the assessment of IOP by Goldmann tonometry, BCVA, anterior chamber, vitreal and retinal statuses. Surgeries took place between October 2002 and May 2010. Sex, age at surgery, preoperative findings, associated diseases, length of follow-up and postoperative complications were documented. Complications included IOP increase, CME, secondary cataract and CCS. The BCVA was determined by the use of Early Treatment Diabetic Retinopathy Study (ETDRS) charts, converted into logarithm of the minimal angle of resolution (LogMAR) units and recorded at the immediate preoperative visit and postoperative follow-up examinations.

\section{PERIOPERATIVE MANAGEMENT}

Preoperatively patients received antibiotics (fluoroquinolones four times daily) and corticosteroids $(0.1 \%$ dexamethasone gel four times daily) at least $24 \mathrm{~h}$ prior to surgery. Postoperatively the medications were continued for up to 2 weeks or tapered, respectively.

\section{STATISTICAL ANALYSIS}

The analysis was undertaken using the commercial software (JMP V.9.0). Results have been presented, with 95\% CIs. Nominal $\mathrm{p}$ values were calculated using t test and the level of significance was set at 0.05 .

\section{RESULTS AND PATIENT CHARACTERISTICS}

The mean age at surgery of our 29 male and 23 female patients was 53 years and ranged between 21 and 89 years. The overall mean follow-up time was 26 months (range 3-60 months). In group 1 the mean follow-up period was 28 months (range 3-52 months) and in group 2, it was 24 months (range 3-60 months). The most common complication was secondary cataract occurring in $44 \%$ (23 eyes) followed by increased IOP (requiring treatment, 10\%; 5 eyes), CCS (4\%, 2 eyes) and $\mathrm{CME}$ (4\%, 2 eyes). Group 1 exhibited 13 eyes $(50 \%)$ with secondary cataract, 4 eyes $(15 \%)$ with IOP elevation, and 2 eyes $(8 \%)$ with CCS and CME, respectively. Group 2 displayed 10 eyes $(38 \%)$ with secondary cataract and 1 (4\%) with CME and elevated IOP, respectively.

\section{Visual outcome}

The group exhibited 19 eyes $(37 \%)$ with unchanged BCVA, 6 eyes (12\%) with a decrease and 27 eyes (52\%) with an increase of the BCVA. The mean preoperative LogMAR BCVA in the entire group was $1.45 \pm 0.85$ (95\% CI 1.21 to 1.69$)$ and increased to $1.32 \pm 0.95$ (95\% CI 1.06 to $1.58, \mathrm{p}=0.02$ ). The mean preoperative and the

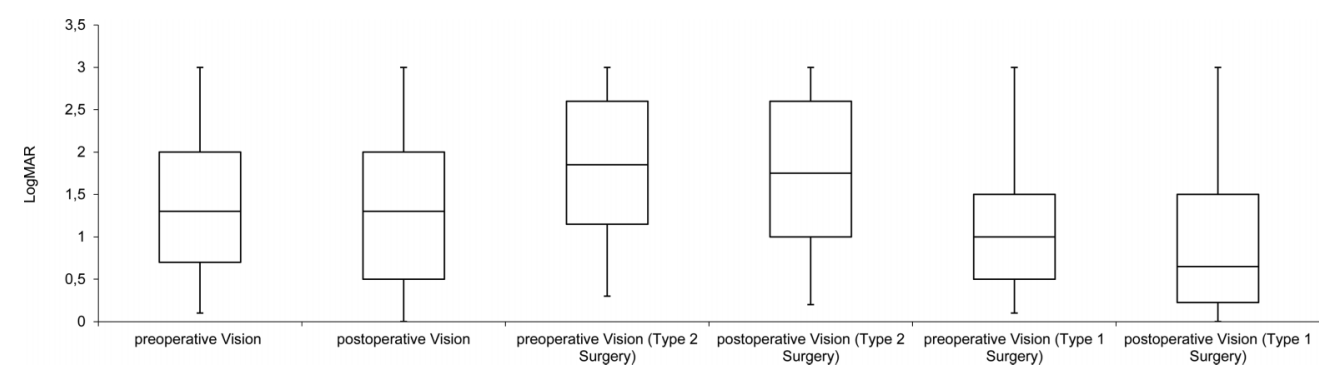

Figure 1 Preoperative and postoperative vision of the logarithm of the minimal angle of resolution (LogMAR) for the total group, types 2 and 1 surgeries. 


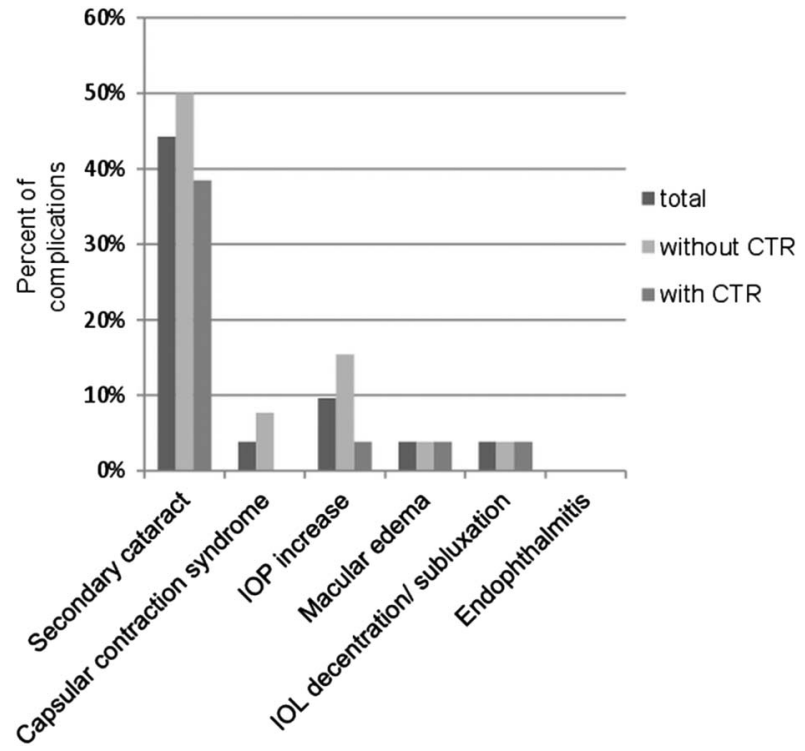

Figure 2 Overview of the postoperative complications.

mean postoperative LogMAR BCVA in the non-CTR group improved from $1.16 \pm 0.8$ (95\% CI 0.83 to 1.48 ) to $0.98 \pm 0.88(95 \%$ CI 0.62 to $1.33, \mathrm{p}=0.02)$ and in the CTR group from $1.74 \pm 0.81$ (95\% CI 1.42 to 2.07) to $1.66 \pm 0.90(95 \%$ CI 1.3 to $2.03, \mathrm{p}=0.31)$, respectively. The preoperative and postoperative overviews are shown in figure 1.

\section{Complications}

The postoperative complications included secondary cataract, increased IOP, CCS and CME in order of occurrence. An overview of the postoperative complications is given in figure 2. In comparison, secondary cataract was seen in $50 \%$ of cases in group 1 and $38 \%$ of the cases in group 2. Concerning CCS all cases were observed in group 1 and no cases in group 2. The increase in IOP was noted in $15 \%$ of cases in group 1 and $4 \%$ of cases in group 2. During the follow-up period one patient of

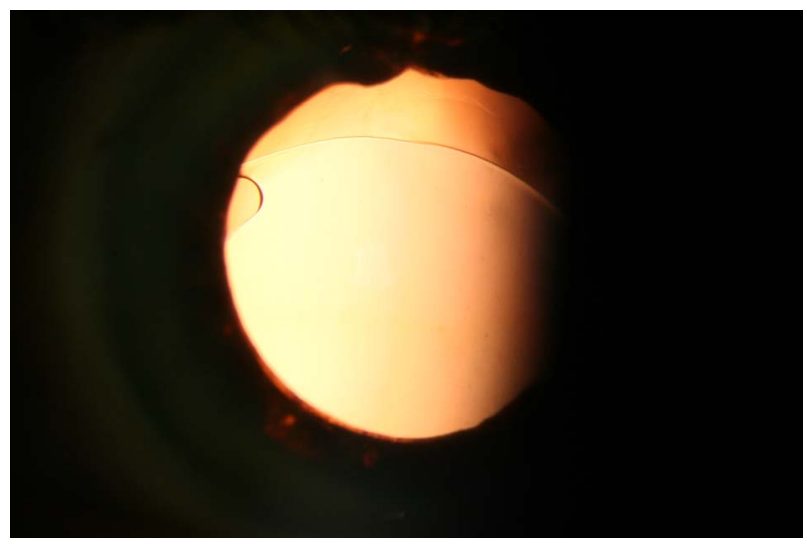

Figure 3 Demonstrates the intraocular lens (IOL) dislocation of one patient with bilateral severe zonular insufficiency.

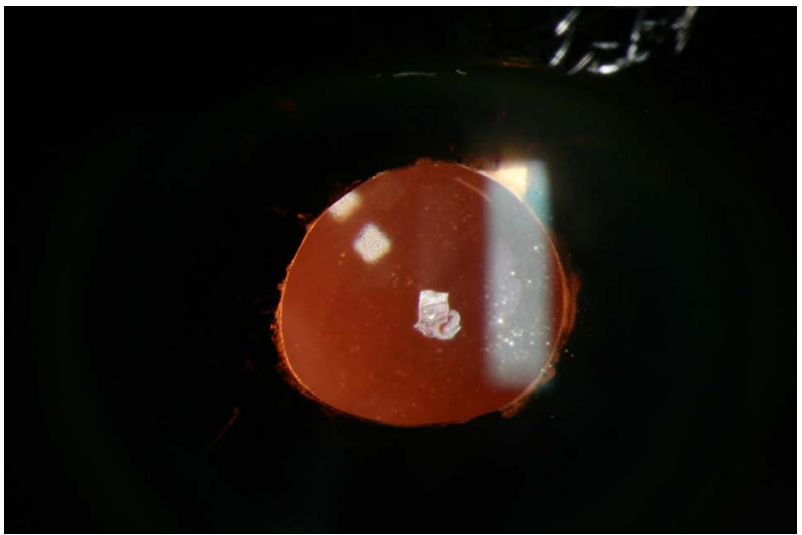

Figure 4 Scleral-fixed IOL of one patient with bilateral severe zonular insufficiency.

group 1 developed subluxated IOL on her right eye and several years later on her left eye. Explantation and implantation of a scleral-fixed lens was performed (figures 3 and 4). A decreased rate of secondary cataract and CCS was noted in group 2. Secondary cataract was seen in 10 eyes in group 2 and 13 eyes in group 1 $(p=0.4)$. CCS was only seen in the group which received type 1 surgery (figure $5 ; \mathrm{p}=0.15$ ).

\section{DISCUSSION}

Cataract is a common complication in patients with RP. The common form is the posterior subcapsular cataract found among all genetic subtypes. ${ }^{3}{ }^{4}$ Fortunately the treatment is straightforward and involves cataract surgery. This procedure may be of great benefit to the patient, keeping in mind the inferior retinal function (figure 6). An improvement of the quality of life is a consequence. Nevertheless, the potential postoperative complications may be frustrating. ${ }^{6}$ To address these, additional implantable devices were conceived. ${ }^{5}$ Whether to utilise them remains up to the surgeon's preference. This retrospective study tried to compare the BCVA and

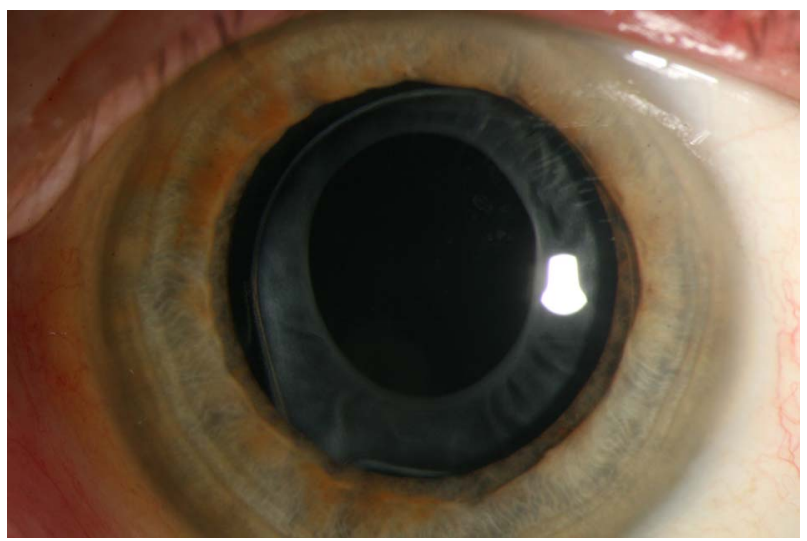

Figure 5 Depicts the developed capsular phimosis in a patient belonging to group 1 , which did not receive additional capsular tension ring insertion. 


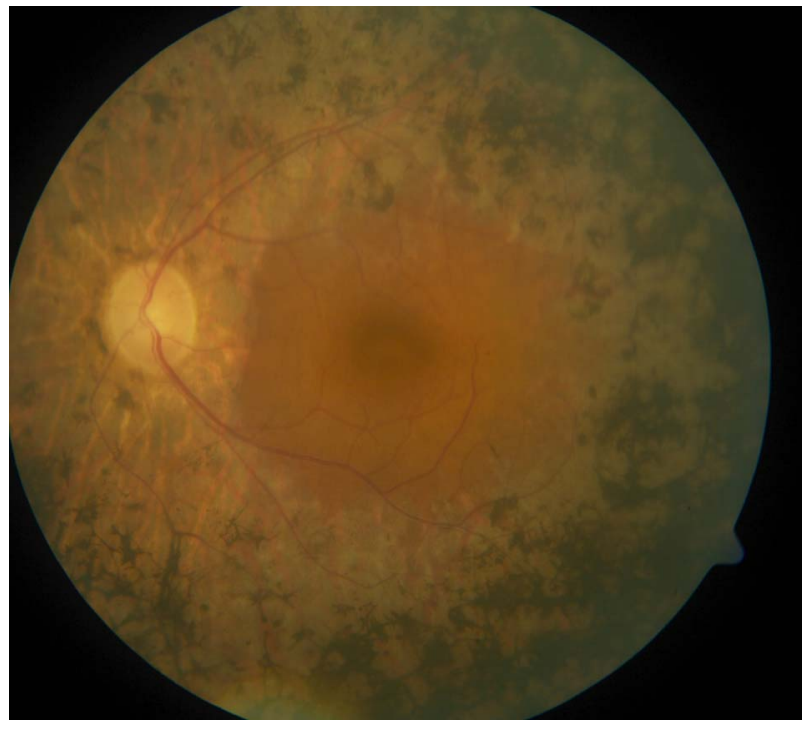

Figure 6 Pathognomonic retinal changes in a retinitis pigmentosa patient.

the development of short-term and long-term postoperative complications after cataract surgery with and without the implantation of a CTR. Type 1 surgery included phacoemulsification and the implantation of an IOL. Type 2 surgery adds a CTR to the inserted IOL. Postoperative complications taken into consideration were IOP, CME, secondary cataract and CCS. ${ }^{3}{ }^{4-14}$ The most common complication was secondary cataract occurring in $44 \%$ (23 eyes) followed by increased IOP (10\%, 6 eyes), CCS (4\%, 2 eyes) and CME (4\%, 2 eyes). Comparing both groups less cases of secondary cataract and CCS could be observed in group 2. The mean preoperative and mean postoperative LogMAR BCVA improved in both groups, although in group 2 the difference was not statistically significant. Group 1 performed better in terms of the final vision. This might be explained by the decreased, preoperative visual acuity of group 2. Thus, both surgical approaches had beneficial outcomes for our patients in terms of a low rate of postoperative complications or increased visual acuity. The decreased number of eyes requiring IOP-lowering therapy in group 2 was considered a coincidence. Five eyes received antiglaucoma therapy. We had no straightforward explanation for this observation. In one case with bilateral subluxated IOL severe zonular insufficiency, noted intraoperatively, was suspected as the cause. ${ }^{14-16}$

The additional implantation of a CTR may prevent IOL decentralisation/subluxation, posterior capsule opacification and anterior capsular phimosis as reported by other groups. ${ }^{15} 17 \quad 18$ A decrease of posterior capsular opacification was also observed. Rapid capsular phimosis may especially occur in patients with RP. ${ }^{19}$ Precautions like CTRs should therefore be considered. ${ }^{5} 18$ Ahmed and colleagues reported improvements in CTR technology enhancing the safety and efficiency in eyes with profound zonular instability. In addition scleral-fixed devices may aid even more in decreasing intraoperative and postoperative risks. ${ }^{5}$ Nevertheless, there are also reports in the literature stating fibrotic reactions, regarding $\mathrm{PCO}$ and $\mathrm{CCS}$, and dislocations in patients with zonular weakness. ${ }^{20-22}$

The limitations of the current study may be the relatively small sample size of this special subset of cataract patients. Further studies may try to include more cases or an analysis of the different genetic subtypes of RP. It may be concluded that less cases of secondary cataract and CCS could be observed in group 2 and that both surgical approaches had beneficial outcomes for our patients in terms of increased visual acuity or decreased postoperative complications.

Contributors TB contributed to the acquisition of data, cleaned and analysed the data, wrote the statistical analysis plan, drafted and revised the manuscript and was involved in the final approval. EY conceptualised the project, monitored data collection, drafted and revised the manuscript and was involved in the final approval of the manuscript. KUBS contributed to the conception, revised the drafted manuscript and was involved in the final approval of the manuscript.

Funding This research received no specific grant from any funding agency in the public, commercial or not-for-profit sectors.

Competing interests None.

Patient consent Obtained.

Ethics approval Obtained.

Provenance and peer review Not commissioned; externally peer reviewed.

Data sharing statement No additional data are available.

\section{REFERENCES}

1. Cruciani F, Amore F, Albanese G, et al. Investigation about causes of blindness and low vision among members of Blind and Visually Impaired Italian Union (UICI). Clin Ter 2011;162:35-42.

2. Wetzel C, Auffarth GU, Krastel H, et al. [Improving contrast sensitivity by cut-off filters in high adaptation luminance levels in retinitis pigmentosa]. [Article in German]. Einfluss von Kantenfiltern auf die Kontrastempfindlichkeit bei Retinitis pigmentosa. Ophthalmologe 1996;93:456-62.

3. Auffarth GU, Nimsgern C, Tetz MR, et al. [Increased cataract rate and characteristics of $\mathrm{Nd}$ :YAG laser capsulotomy in retinitis pigmentosa]. [Article in German]. Erhoehte Nachstarrate und Besonderheiten der Nd:YAG-Laserkapsulotomie bei Retinitis pigmentosa. Ophthalmologe 1997;94:791-5.

4. Auffarth GU, Tetz MR, Krastel $\mathrm{H}$, et al. [Complicated cataracts in various forms of retinitis pigmentosa. Type and incidence]. [Article in German]. Cataracta complicata bei verschiedenen Formen der Retinitis pigmentosa Art und Häufigkeit. Ophthalmologe 1997;94:642-6.

5. Hasanee K, Butler M, Ahmed II. Capsular tension rings and related devices: current concepts. Curr Opin Ophthalmol 2006;17:31-41.

6. Najjar DM, Igbre AO, Tsai FF. Late capsular bag contraction and intraocular lens subluxation in retinitis pigmentosa: a case report. $J$ Med Case Reports 2011;5:65.

7. Lam FC, Livingstone I, Imrie FR, et al. Delayed dislocation of an injectable hydrophilic acrylic lens after Nd:YAG capsulotomy in anterior capsular contraction syndrome. Cont Lens Anterior Eye 2011;34:193-5.

8. Cheour M, Brour J, Boukari M, et al. Capsulorhexis contraction syndrome despite capsular tension ring implantation. Bull Soc Belge Ophtalmol 2010;315:19-21.

9. Panagopoulos A, Chalioulias K, Kirkby GR. A new approach in the surgical management of anterior capsular phimosis syndrome. Ophthalmic Res 2009;42:221-3.

10. Nagy ZZ, Ecsedy M, Kovács I, et al. Macular morphology assessed by optical coherence tomography image segmentation after femtosecond laser-assisted and standard cataract surgery. $J$ Cataract Refract Surg 2012;38:941-6. 
11. Scarpa G. Bilateral cystoid macular edema after cataract surgery resolved by vitrectomy. Eur J Ophthalmol 2011;21:677-9.

12. Mansberger SL, Gordon MO, Jampel $\mathrm{H}$, et al. Ocular Hypertension Treatment Study Group. Reduction in intraocular pressure after cataract extraction: the Ocular Hypertension Treatment Study. Ophthalmology 2012;119:1826-31.

13. Su WW, Chen PY, Hsiao $\mathrm{CH}$, et al. Primary phacoemulsification and intraocular lens implantation for acute primary angle-closure. PLOS ONE 2011;6:20056.

14. Lee HJ, Min SH, Kim TY. Bilateral spontaneous dislocation of intraocular lenses within the capsular bag in a retinitis pigmentosa patient. Korean J Ophthalmol 2004;18:52-7.

15. Takimoto M, Hayashi K, Hayashi $\mathrm{H}$. Effect of a capsular tension ring on prevention of intraocular lens decentration and tilt and on anterior capsule contraction after cataract surgery. Jpn J Ophthalmol 2008;52:363-7.

16. Davis D, Brubaker J, Espandar L, et al. Late in-the-bag spontaneous intraocular lens dislocation: evaluation of 86 consecutive cases. Ophthalmology 2009;116:664-70.
17. D'Eliseo D, Pastena B, Longanesi L, et al. Prevention of posterior capsule opacification using capsular tension ring for zonular defects in cataract surgery. Eur J Ophthalmol 2003;13:151-4.

18. Hasanee K, Ahmed II. Capsular tension rings: update on endocapsular support devices. Ophthalmol Clin North Am 2006;19:507-19.

19. Nikpoor N, Stone DU. Rapid capsular phimosis in retinitis pigmentosa. Ophthalmic Surg Lasers Imaging 2010. Epub ahead of print 29 Jul 2010. doi: 10.3928/15428877-20100726-12.

20. Masket S, Osher R. Late complications with intraocular lens dislocation after capsulorhexis in pseudoexfoliation syndrome. J Cataract Refract Surg 2002;28:1482-4.

21. Sudhir R, Rao S. Capsulorhexis phimosis in retinitis pigmentosa despite capsular tension ring implantation. J Cataract Refract Surg 2001:27:1691-4.

22. Moreno-Montañés J, Sánchez-Tocino H, Rodriguez-Conde R. Complete anterior capsule contraction after phacoemulsification with acrylic intraocular lens and endocapsular ring implantation. $J$ Cataract Refract Surg 2002;28:717-19. 